\title{
Optic Pit Maculopathy: Adjunctive Treatment Using Oral Spironolactone and Topical Dorzolamide
}

\section{Susan Ruyu Qi \\ Eunice You (iD \\ Melanie Hebert (D) \\ Ali Dirani (D)}

Department of Ophthalmology, Hôpital du Saint-Sacrement, Centre Hospitalier Universitaire de Québec - Université Laval, Québec City, Québec, Canada
Correspondence: Ali Dirani Centre Universitaire d'Ophtalmologie, Hôpital du Saint-Sacrement, Centre Hospitalier Universitaire de Québec Université Laval, Québec City, Québec, Canada

Email drdirani@gmail.com
Purpose: There is no consensus for the treatment of optic disc pit maculopathy (ODPM). We describe a case of ODPM refractory to vitrectomy that was successfully treated with systemic spironolactone and topical dorzolamide $2 \%$.

Methods: Case report.

Results: A 27-year-old male was referred for decreased vision in the right eye secondary to macular edema. Fundus examination and optical coherence tomography imaging revealed an optic pit maculopathy with significant macular schisis and intraretinal fluid. After an initial observation period, we performed pars plana vitrectomy with internal limiting membrane peeling, fibrin glue, and gas endotamponade. At the 2 months postoperative follow-up, anatomic and functional outcomes remained unimproved. Oral spironolactone and topical dorzolamide $2 \%$ were, therefore, added. Significant reduction in the intraretinal fluid and macular schisis was demonstrated at the last follow-up 2 years postoperative.

Conclusion: The mechanisms underlying optic disc pit maculopathy remain controversial, and treatment guidelines are unclear. To our knowledge, this is the first description of an OPDM treated with a combination systemic mineralocorticoid receptor antagonist and topical carbonic anhydrase inhibitor as adjuvant therapy to vitrectomy with favorable outcomes. We also review the literature and propose mechanisms through which these treatment entities may act.

Keywords: dorzolamide, optic disc pit maculopathy, spironolactone

\section{Summary Statement}

We report the case of a 27-year-old male with optic disc pit maculopathy associated with macular schisis and intraretinal fluid. Pars plana vitrectomy with gas tamponade, internal limiting membrane peeling, and fibrin glue was performed. Adjuvant oral spironolactone and topical dorzolamide $2 \%$ therapy resulted in anatomic and functional improvement 2 years postoperatively.

\section{Introduction}

Optic disc pit (ODP) is a rare congenital abnormality characterized by small, hypopigmented, and excavated colobomatous defects of the optic nerve head. While most OPD are asymptomatic, a subset of patients may present with serous detachment and/or retinoschisis of the central macula. This complication, referred to as optic disc pit maculopathy (ODPM), is characterized by an accumulation of 
subretinal or intraretinal fluid (SRF/IRF) at the macula and may result in poor visual prognosis if left untreated. ${ }^{1,2}$

The origin of this fluid remains up for debate but is thought to originate from the vitreous or the cerebrospinal fluid (CSF). Currently, there is still no clear consensus on the most effective treatment of ODPM. ${ }^{1}$ Pars plana vitrectomy (PPV) is widely considered to be the preferred treatment modality, and a number of other adjunctive therapies have been employed including endolaser, gas tamponade, inner limiting membrane (ILM) peeling, SRF drainage, fibrin glue, and/or autologous platelet injections. ${ }^{2}$ While a quarter of cases may resolve spontaneously, surgical intervention is still the recommended approach due to high risk of recurrence and poor visual outcome. ${ }^{1}$ In this report, we describe a case of ODPM refractory to PPV that was successfully treated with systemic spironolactone combined with a topical carbonic anhydrase inhibitor (CAI). We therefore propose spironolactone and CAI as adjuvant therapy in the treatment of ODPM and suggest possible mechanisms through which these agents may induce resorption of subretinal fluid after surgery.

\section{Case Report}

A healthy 27-year-old male was referred by his optometrist for decreased vision in the right eye secondary to macular edema. On examination, visual acuity was $20 / 40$ in the right eye and dilated fundus examination revealed an optic pit located at the inferotemporal border of the optic nerve. Optical coherence tomography (OCT) imaging of the right eye revealed the presence of macular schisis associated with significant IRF (Figure 1). The left eye was normal. The patient was observed for 5 months with no anatomic or functional improvement to the OPDM. We therefore decided to intervene surgically. The patient underwent a PPV with ILM peeling at the macula and around the optic nerve, endolaser photocoagulation at the edge of optic pit, $25 \%$
$\mathrm{SF}_{6}$ gas tamponade followed by injection of fibrin glue (ARTISS, Baxter International Inc: Vienna, Austria). At the 2-month postoperative follow-up, VA was measured at 20/50 and OCT remained unchanged, with unchanged IRF and macular thickening. Given the lack of improvement, we decided to place the patient on oral spironolactone $25 \mathrm{mg}$ twice daily and topical dorzolamide $2 \%$ drops thrice daily. At the 10-month follow-up, OCT demonstrated a significant reduction in IRF. Oral spironolactone was pursued and at the latest follow-up 2 years post-operative, there was further improvement of the OCT as well as VA to 20/30 (Figure 2). The patient provided informed consent for the publication of their case details and any accompanying images. Institutional approval was not required to publish the case details.

\section{Discussion}

Optic disc pits (ODP) are rare entities. Visual deterioration occurs only when ODP are associated with maculopathy, which occurs in approximately 25 to $75 \%$ of cases. ${ }^{1}$ The mechanism of ODPM remains poorly understood and optimal management controversial, including treatment, timing, and long-term outcomes. ${ }^{1,2} \mathrm{~A}$ recent review by Bloch et al suggests that a conservative approach with initial observation may be preferable as delaying surgery does not convey a poorer outcome. ${ }^{3}$ Other sources have suggested that although a quarter of OPDM cases may resolve spontaneously, a proactive surgical approach is recommended as visual outcome tends to be poor in untreated cases, with gradual worsening and a final VA of 20/200 or worse. ${ }^{1}$

When an intervention is deemed necessary, PPV is widely considered to be the mainstay treatment. ${ }^{1} \mathrm{PPV}$ is often combined with laser treatment, gas tamponade, and/ or ILM peeling. Other techniques have also been described including macular buckling, inner retinal fenestration, autologous fibrin and glial tissue removal. ${ }^{1,2}$ In a long-
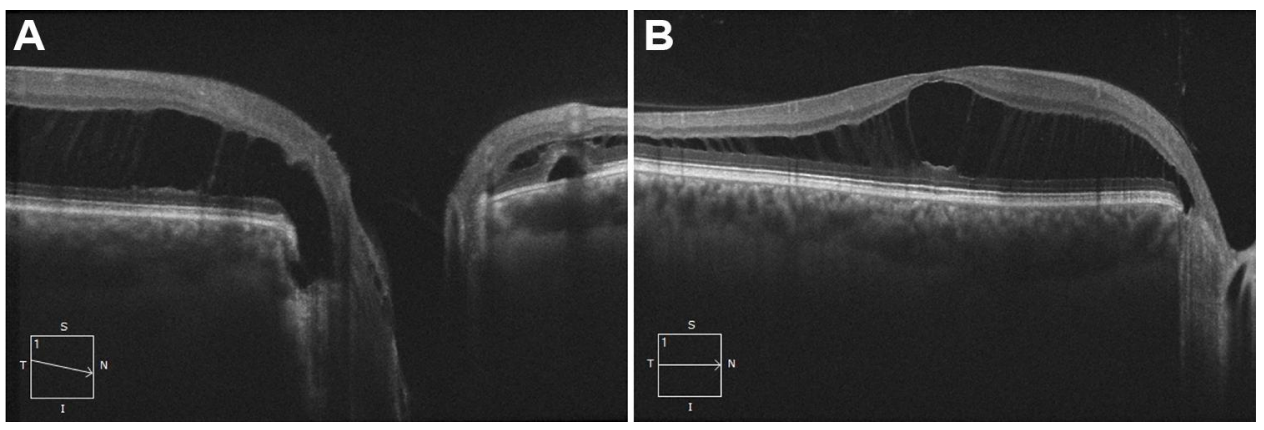

Figure I Optical coherence tomography imaging of the patient's right eye on initial presentation, demonstrating (A) an inferotemporal optic nerve pit with retinoschisis, intraretinal fluid, subretinal fluid, and (B) significant macular schisis. 

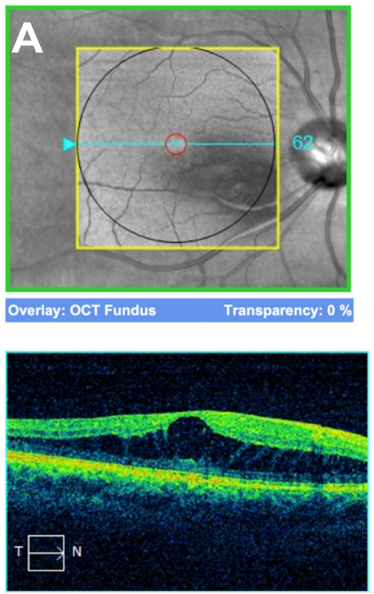
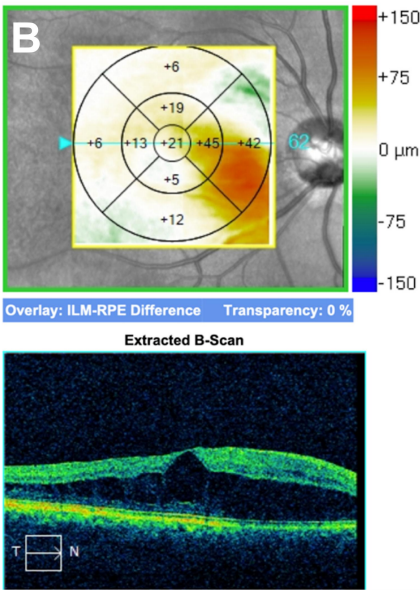
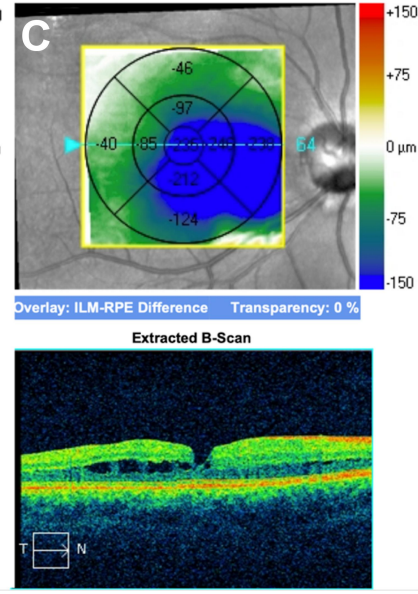
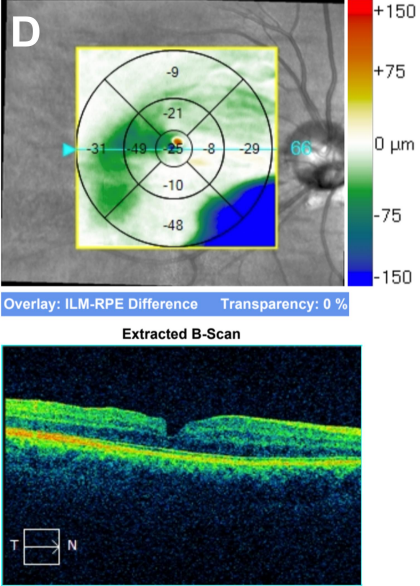

Figure 2 Optical coherence tomography macular change analysis showing structural evolution of the macula (A) at initial presentation, (B) 2 months post-operatively with persistence of macular schisis, (C) 10 months post-operatively with reduction of the intraretinal fluid following adjunctive use of oral spironolactone and topical dorzolamide and (D) 2 years post-operatively with almost complete resolution of the intraretinal fluid following long-term spironolactone use.

term case series, vitrectomy for optic pit maculopathy has been demonstrated to be a safe and successful therapeutic option with complete resolution of fluid achieved in 8 out of 10 eyes without additional treatment. ${ }^{4}$ However, there is no consensus on medical treatment options, or adjunctive steps that could be taken following vitrectomy in patients with poor surgical outcomes. ${ }^{2}$ An individualized approach is recommended. ${ }^{1}$

The origin of fluid in ODPM is unclear. Previous studies have all failed to demonstrate a clear pathogenesis for this condition. ${ }^{1}$ Some have suggested that this fluid originates from the vitreous or the CSF while others have shown that there are leaking vessels around the ODP. ${ }^{1}$ Spironolactone was started in our patient based on the results of previous studies in which SRF and IRF reduction was achieved using mineralocorticoid receptor (MR) antagonists for central serous retinopathy (CSR). ${ }^{5} \mathrm{MR}$ creates choroidal vasodilation and leakage, which leads to choroidal hyperpermeability. ${ }^{6}$ Therefore, MR antagonists, such as spironolactone and eplerenone, are hypothesized to induce the resorption of retinal fluid by decreasing vascular hyperpermeability. ${ }^{6}$

Dorzolamide, on the other hand, is a topical CAI. These inhibitors are thought to interact with carbonic anhydrase enzymes present in the retinal pigment epithelium (RPE) and Müller cells. By increasing RPE pump and Müller cell activity, there is increased subretinal fluid absorption from the retina to the choroid. Previous reports have suggested CAI for X-linked retinoschisis, ${ }^{7}$ choroideremia, ${ }^{8}$ and CSR. ${ }^{9}$ Recently, a case described the resolution of IRF accumulation in an ODP-associated macular retinoschisis following topical dorzolamide $2 \%$ thrice daily. Over two years, vision improved to 20/20 with improvement of the macular retinoschisis. ${ }^{10}$

In conclusion, due to the limited sample sizes of case series and paucity of comparative studies regarding ODPM, it is difficult to fully elucidate its physiopathology and subsequently counsel patients on the best treatment options. This is the first case report in which a combined approach of systemic spironolactone and topical carbonic anhydrase inhibitor was used as adjunctive treatment. We report notable reduction in the IRF and SRF, with seemingly improved final VA in our patient. While it is unclear how much of the improvement in anatomic and functional outcomes is attributable to the addition of spironolactone and dorzolamide compared to spontaneous resolution or a beneficial response to the surgical intervention, which can occur up to two years after surgery, we believe that there may be a pathophysiologic basis for the use of adjunctive systemic mineralocorticoid antagonists and topical carbonic anhydrase inhibitors that is worth further investigating. The pathophysiology of central serous retinopathy also differs from optic disc pit maculopathy pathogenesis, but both are characterized by subretinal and intraretinal fluid and may therefore benefit from the action of an aldosterone antagonist on vascular permeability. Furthermore, the patient continues to be on spironolactone, which may play a role in stabilizing and continuing to improve visual function as previously described.

At this time, our case report does not allow us to make further conclusions and further studies with control groups 
are needed to confirm our findings. Investigating adjunctive medical treatment in patients that do not undergo surgical intervention (eg due to patient preference or acceptable functional outcomes at baseline) or in patients that do not respond to surgical intervention at two years would also allow us to better elucidate any beneficial treatment effect from the use of these agents.

\section{Disclosure}

This manuscript has not been presented at a conference or meeting prior to submission. This manuscript is original, has not been published before and is not currently being considered for publication elsewhere. No conflicts of interest were declared for any author.

\section{References}

1. Kalogeropoulos D, Ch'ng SW, Lee R, et al. Optic disc pit maculopathy: a review. Asia Pac J Ophthalmol. 2019;8(3):247-255. doi:10.22608/APO.2018473

2. Chatziralli I, Theodossiadis P, Theodossiadis GP. Optic disk pit maculopathy: current management strategies. Clin Ophthalmol. 2018;12:1417-1422. doi:10.2147/OPTH.S153711
3. Bloch E, Georgiadis O, Lukic M, da Cruz L. Optic disc pit maculopathy: new perspectives on the natural history. Am J Ophthalmol. 2019;207:159-169. doi:10.1016/j.ajo.2019.05.010

4. Bottoni F, Cereda M, Secondi R, Bochicchio S, Staurenghi G. Vitrectomy for optic disc pit maculopathy: a long-term follow-up study. Graefes Arch Clin Exp Ophthalmol. 2018;256(4):675-682. doi:10.1007/s00417-018-3925-9

5. Daruich A, Matet A, Dirani A, et al. Central serous chorioretinopathy: recent findings and new physiopathology hypothesis. Prog Retin Eye Res. 2015;48:82-118. doi:10.1016/j.preteyeres.2015.05.003

6. Zhao M, Célérier I, Bousquet E, et al. Mineralocorticoid receptor is involved in rat and human ocular chorioretinopathy. $J$ Clin Invest. 2012;122(7):2672-2679. doi:10.1172/JCI61427DS1

7. Ajlan RS, Hammamji KS. Stellate nonhereditary idiopathic foveomacular retinoschisis: response to topical dorzolamide therapy. Retin Cases Brief Rep. 2019;13(4):364-366. doi:10.1097/ICB.0000000 000000599

8. Genead MA, McAnany JJ, Fishman GA. Topical dorzolamide for treatment of cystoid macular edema in patients with choroideremia. Retina. 2012;32(4):826-833. doi:10.1097/IAE.0b013e3182215ae9

9. Das D, Nigam E. Resolution of cystoid macular edema by topical dorzolamide in a case of central serous chorioretinopathy: a case report. Med Vis Res Found. 2017;XXXV(3):25-26.

10. Al-Moujahed A, Callaway NF, Vail D, Ludwig CA, Ji MH, Moshfeghi DM. Resolution of optic disc pit-associated macular retinoschisis after topical carbonic anhydrase inhibitor treatment: report of a case. Eur J Ophthalmol. 2020:112067212090466. doi:10.1177/1120672120904664

\section{Publish your work in this journal}

The International Medical Case Reports Journal is an international, peer-reviewed open-access journal publishing original case reports from all medical specialties. Previously unpublished medical posters are also accepted relating to any area of clinical or preclinical science. Submissions should not normally exceed 2,000 words or 4 published pages including figures, diagrams and references. The manuscript management system is completely online and includes a very quick and fair peer-review system, which is all easy to use. Visit http://www.dovepress.com/testimonials.php to read real quotes from published authors. 